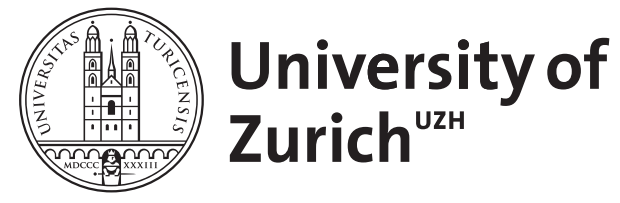
Archive

University of Zurich

University Library

Strickhofstrasse 39

CH-8057 Zurich

www.zora.uzh.ch

Year: 2014

\title{
Positionen aktueller Mohammed-Forschung
}

Rudolph, Ulrich ; Uehlinger, Christoph

DOI: https://doi.org/10.1515/asia-2014-0031

Posted at the Zurich Open Repository and Archive, University of Zurich

ZORA URL: https://doi.org/10.5167/uzh-99253

Journal Article

Published Version

Originally published at:

Rudolph, Ulrich; Uehlinger, Christoph (2014). Positionen aktueller Mohammed-Forschung. Asiatische Studien, 68(2):433-437.

DOI: https://doi.org/10.1515/asia-2014-0031 
2014 - VOLUME 68 - NUMBER 2

\section{ASIATISCHE STUDIEN ÉTUDES ASIATIQUES \\ ZEITSCHRIFT DER SCHWEIZERISCHEN \\ ASIENGESELLSCHAFT REVUE DE LA SOCIÉTÉ SUISSE-ASIE}

EDITOR OF THIS ISSUE

Maurus Reinkowski, Basel

EDITOR-IN-CHIEF

Rafael Suter, Zürich

\section{DE GRUYTER}


EDITOR-IN-CHIEF Rafael Suter, Asien-Orient-Institut, Universität Zürich, Zürichbergstrasse 4, CH-8032 Zürich, E-mail: sag.editor@aoi.uzh.ch

EDITORIAL BOARD Blain Auer, Lausanne. Norman Backhaus, Zürich. Wolfgang Behr, Zürich. Daria Berg, St. Gallen. Maya Burger, Lausanne. David Chiavacci, Zürich. Bettina Dennerlein, Zürich. Karénina Kollmar-Paulenz, Bern. Anke von Kügelgen, Bern. Angelika Malinar, Zürich. Annemarie Mertens, Zürich. Silvia Naef, Genève. Maurus Reinkowski, Basel. Andrea Riemenschnitter, Zürich. Ulrich Rudolph, Zürich. Reinhard Schulze, Bern. Pierre Souyri, Genève. Raji C. Steineck, Zürich. Ingo Strauch, Lausanne. Christoph Uehlinger, Zürich. Nicolas Zufferey, Genève.

Publiziert mit Unterstützung der Schweizerischen Akademie der Geistes- und Sozialwissenschaften. Publiée avec le soutien de l'Académie suisse des sciences humaines et sociales.

Published with the support of the Swiss Academy of Humanities and Social Sciences. 


\section{Inhaltsverzeichnis - Table des Matières - Contents}

\section{Aufsätze - Articles - Articles}

Ulrich Rudolph und Christoph Uehlinger

Positionen aktueller Mohammed-Forschung — 433

Fred M. Donner

Muhammad und die frühe islamische Gemeinschaft aus historischer

Sicht -439

Tilman Nagel

Der Weg zum geschichtlichen Mohammed - 453

Gregor Schoeler

Tilman Nagels „,Authentizität‘ in der Leben-Mohammed-Forschung“. Eine Antwort -469

Andreas Görke und Harald Motzki

Tilman Nagels Kritik an der Isnad-cum-matn-Analyse. Eine Replik — 497

Hussein Ali Abdulsater

To rehabilitate a theological treatise. Inqādh al-Bashar min

al-Jabr wa-l-Qadar -519

Florian Zemmin

Was hat Max Weber mit Kātib Čelebi zu tun? Ein Annäherungsversuch an Gottfried Hagen — 549

Georges Tamer

Den Orient begrifflich denken: Überlegungen zur Erforschung der arabischislamischen Geistesgeschichte -557

Ewald Wagner

Verse über Verse: Wie Ibn al-Ḥağğāğ seine eigene Poesie betrachtet — 579 


\section{Rezensionen - Comptes rendus - Reviews}

Karénina Kollmar-Paulenz

Arslan, Saadet. Tibetische Handschriften und Blockdrucke - 619

Karénina Kollmar-Paulenz

Bawden, Charles R. Another Tract for the Buryats with I.J. Schmidt's recently identified Kalmuck originals — 623

Sophie Glutz von Blotzheim Bürgel, Johann Christoph. Liebesrausch und Liebestod in der islamischen Dichtung des 7.-15. Jahrhunderts — 627

Martin Lehnert

Paul, Gregor / Schneider, Elisabeth / Paul, Irene (Hg.). Das große Lexikon des Buddhismus, zweite Lieferung: Bait - D—633

Stefan Georg

Knüppel, Michael. Sprachtabus in tungusischen Sprachen und Dialekten. Am Beispiel von S.M. Širokogorovs „Tungus Dictionary“— 641

Chiara Formichi

Laffan, Michael. The Makings of Indonesian Islam: Orientalism and the Narration of a Sufi Past — 657

Martin Lehnert

Radich, Michael. How Ajātaśatru Was Reformed: The Domestication of "Ajase" and Stories in Buddhist History — 661 


\section{Ulrich Rudolph und Christoph Uehlinger Positionen aktueller Mohammed-Forschung}

DOI 10.1515/asia-2014-0031

Die Person Mohammeds, sein Wirken und seine historische Bedeutung waren und sind immer wieder Gegenstand kontroverser Stellungnahmen. Dabei kommen nicht nur unterschiedliche Wahrnehmungen und Einschätzungen zum Ausdruck, sondern auch eine Pluralität von Fragestellungen und (Erkenntnis-) Interessen, die sich verschiedenen Diskursen zuordnen lassen.

Einer dieser Diskurse ist der im engeren Sinne wissenschaftliche - womit hier vor allem die Islamwissenschaft, die Religionswissenschaft und die Geschichtswissenschaft gemeint sind. Er hat seit langem zum Ziel, ein historisch verlässliches Bild von Mohammeds Leben und Wirken zu zeichnen, sieht sich aber immer schon mit der skeptischen Frage konfrontiert, ob man angesichts der problematischen Quellenlage überhaupt etwas Verlässliches darüber wissen kann. Diese Frage wurde in den letzten Jahrzehnten wieder mit besonderer Dringlichkeit gestellt. Nach wie vor besteht, was explizite textliche Hinweise auf Mohammeds Biographie betrifft, kein Konsens darüber, wie die umfangreiche islamische Überlieferung und die spärliche ausserislamische Überlieferung zu seinem Leben zu gewichten seien. Folglich stehen wir noch immer vor grundsätzlichen Fragen wie diesen, um nur einige Beispiele zu nennen: Welche der zahlreichen in den Quellen tradierten Aussagen führen uns zeitlich am nächsten an den Propheten des Islams heran? Welche von ihnen sind die historisch zuverlässigsten (was nicht dasselbe sein muss)? Und welche Methoden eignen sich am besten, um eine Klärung solcher Fragen - wenn sie denn möglich sein sollte - herbeizuführen?

Ein anderer Diskurs ist breiter angelegt und findet vor allem in der Öffentlichkeit allgemeines Interesse. Sein Ziel besteht darin, Mohammeds Persönlichkeit, seine Intentionen, seine Besonderheiten und charakteristischen Ziele zu erfassen, um auf diese Weise Einblicke in die Anfänge des Islams, wenn nicht des Islams überhaupt zu gewinnen. Dieser Erkenntnisanspruch ist weit gefasst und verträgt sich nur schwer mit den Aporien, die sich aus der gerade skizzierten, problematischen Quellenlage ergeben. Gleichwohl ist es legitim, solche

Ulrich Rudolph: Abteilung Islamwissenschaft, Asien-Orient-Institut, Universität Zürich.

E-mail: ulrich.rudolph@aoi.uzh.ch

Christoph Uehlinger: Religionswissenschaftliches Seminar, Universität Zürich.

E-mail: Christoph.Uehlinger@uzh.ch 
Fragen zu stellen, zumal in einer Zeit, da der Islam allenthalben eine gelebte, gesellschaftliche Realität darstellt und eine Verständigung über seine Grundlagen und seine Gründerfigur im allgemeinen Interesse - auch der Muslime - ist. Die Gefahr, hier vorschnelle Schlüsse zu ziehen, ist allerdings immens. Das zeigt sich schon an der enormen Bandbreite von Urteilen und Mutmassungen, die in diesem Zusammenhang geäussert werden. Sie sind nicht immer sachgeleitet, sondern reichen von der Dämonisierung Mohammeds bis hin zu seiner Verklärung, wobei extreme Positionierungen häufig eine besondere Aufmerksamkeit in der Öffentlichkeit finden. Für die historisch-wissenschaftliche Fragestellung sind derlei Darstellungen und Bewertungen allemal bemerkenswert: Die komplexen Verflechtungen von Rekonstruktion und Rezeption verlangen nach methodologischer Reflexion und theoretischer Vertiefung im Sinne einer Diskursgeschichte und einer Geschichte der kulturellen Gedächtnisse. ${ }^{1}$

Das Auseinanderklaffen von Mohammedbildern und -darstellungen, insbesondere in Europa, dürfte ein Grund dafür sein, dass sich in den letzten Jahren ein weiterer Diskurs etabliert hat. Sein Ziel ist es, zwischen den Bemühungen der Wissenschaft und den legitimen Interessen der breiteren Öffentlichkeit zu vermitteln. Das geschieht vor allem durch die Publikation von kurzen, ins Thema einführenden Überblicksdarstellungen, in denen die Ergebnisse der Forschung über Mohammed zusammengefasst, gesichtet und einem breiteren Lesepublikum vorgestellt werden. Solche Darstellungen erfüllen einen mehrfachen Zweck: Sie resümieren scheinbar „verbürgtes“ Wissen (d.h. im Falle Mohammeds: die traditionelle Biographie, die sich aus den islamischen Quellen ergibt und die in vergleichbarer Form lange auch von der Islamwissenschaft und der Religionswissenschaft wiedergegeben wurde) und versuchen gleichzeitig, die methodischen Fragezeichen offen zu legen, die sich daran knüpfen. Darüber hinaus geht es ihnen darum, die verschiedenen Mohammedbilder und -diskurse zu thematisieren, die über Jahrhunderte in der islamischen Welt und im Westen gepflegt wurden. Damit soll der Deutungshorizont offen gelegt werden, in dem sich die Debatten über Mohammed - in welcher Form und mit welchem Anspruch auch immer - bis heute bewegen. ${ }^{2}$

1 Vgl. hierzu etwa Shalem 2013.

2 Wenn man sich auf Publikationen in deutscher Sprache beschränkt, sind hier vor allem drei islamwissenschaftliche Bücher aus den letzten Jahren zu nennen, Bobzin 2011, Schöller 2008 und Brunner 2011; eine Zusammenfassung aus religionswissenschaftlicher Sicht bietet Tworuschka 2000. - Darüber hinaus sind in letzter Zeit mehrere kurze Darstellungen erschienen, die eine Einführung in die Thematik mit Zielsetzungen verbinden, die nicht primär wissenschaftlich begründet, sondern teilweise ideologisch orientiert sind. Prominente Beispiele dafür sind die skeptisch-sarkastischen Ausführungen in Jansen 2008 und die weitgehend apologetische Darstellung in Ramadan 2009. 
Ein vergleichbares Anliegen lag der Vortragsreihe zugrunde, die im Herbstsemester 2013 unter dem Titel „Positionen aktueller Mohammed-Forschung“ an der Universität Zürich stattfand. Sie wurde von den beiden Unterzeichnenden für die Studierenden der Islamwissenschaft und der Religionswissenschaft sowie für ein weiteres interessiertes Publikum veranstaltet und bot fünf prominenten Forschern aus dem englischen, französischen und deutschen Sprachraum, darunter einem islamischen Theologen, die Möglichkeit, ihre Sichtweisen auf Mohammed zu präsentieren. Der Schwerpunkt der Vorträge lag nicht auf den Fragen der Quellenanalyse und der damit verbundenen Methodik. Hauptzweck der Reihe war es vielmehr, verschiedene Möglichkeiten der Kontextualisierung und der Deutung von Mohammeds Person und Wirken (historisch, religionsgeschichtlich, theologisch, politisch usw.) zu diskutieren, was den Interessen der Teilnehmenden entsprach und auch zu lebhaften Diskussionen mit und unter ihnen führte. Im vergleichenden Nebeneinander wurde deutlich, wie sehr das Bild, das ein Autor vom historischen Mohammed zeichnet, nicht nur von Vorentscheidungen bezüglich der Auswahl und der Gewichtung unterschiedlicher Quellen (etwa des Korans oder der Sīra), sondern auch von methodischen Akzentsetzungen (exegetischen, komparatistischen, sozialgeschichtlichen) und von unterschiedlichen Fokussierungen des historiographischen Horizonts abhängt (je nachdem, ob Mohammed im engeren Rahmen der arabischen Halbinsel, Syro-Mesopotamiens oder der sich zwischen Mittelmeer, Westasien und Nordwestafrika erstreckenden spätantiken Welt situiert wird). ${ }^{3}$ Zwei der Vorträge sind auf den folgenden Seiten abgedruckt, nämlich die Beiträge von Fred M. Donner (Chicago) und Tilman Nagel (Göttingen). Beide Autoren gehen die angesprochene Thematik unterschiedlich an; ihnen ist aber gemeinsam, dass sie die Beweggründe und Überlegungen resümieren, denen sie bei ihren jeweiligen Forschungen und Publikationen über Mohammed gefolgt sind. Deswegen konnte in beiden Fällen auf einen ausführlichen Anmerkungsapparat verzichtet werden (der sich in den jeweils zitierten umfangreicheren Publikationen der Autoren natürlich findet) und der Vortragsstil der präsentierten Texte beibehalten werden.

Während die Zürcher Vortragsreihe (zu der neben den Genannten auch Marco Schöller, Claude Gilliot und Mouhanad Khorchide beigetragen haben) also versuchte, einen Einblick in die aktuelle Mohammed-Forschung zu vermitteln, ging eben diese Forschung natürlich weiter. Das dokumentieren die beiden anderen Beiträge, die im Folgenden präsentiert werden und die zum einen von Gregor Schoeler, zum anderen von Andreas Görke und Harald Motzki stammen. Ihr Fokus ist die Debatte über die Methoden, die bei der Analyse des umfangreichen

3 Vgl. hierzu nun Fowden 2014. 
islamischen Überlieferungsmaterials zu Mohammed angewandt werden sollten. Diese Debatte wird nicht nur im deutschen Sprachraum, aber dort besonders intensiv geführt, woran vor allem die drei genannten Autoren sowie Tilman Nagel beteiligt sind. Gregor Schoeler hatte bereits 2011 in den Asiatischen Studien einen kritischen Besprechungsaufsatz zu Tilman Nagels Buch Mohammed. Leben und Legende veröffentlicht. ${ }^{4}$ Nagel antwortete darauf 2013 in der Zeitschrift Arabica mit einer ausführlichen Replik, in der er nicht nur die Arbeiten von Schoeler, sondern auch jene von Harald Motzki und Andreas Görke kritisierte. ${ }^{5}$ Darauf reagieren nun wieder Schoeler, Motzki und Görke in den in diesem Heft abgedruckten Aufsätzen, indem sie sich kritisch mit Nagels Einwänden gegen ihre Publikationen auseinandersetzen.

Die folgenden vier Beiträge gehören also unterschiedlichen Textgattungen an. Hinzu kommt, dass Schoeler, Motzki und Görke zwar Nagel ausführlich kritisieren, sich aber auf seinen Beitrag in Arabica und nicht auf den Vortragstext beziehen, der hier erst veröffentlicht wird. Gleichwohl ist das Nebeneinander der verschiedenen Beiträge ertragreich. Es zeigt nämlich, wie brisant die gesamte Thematik ist und wie viele Optionen und Perspektiven geprüft werden müssen, wenn die Forschungen über Mohammed voranschreiten sollen. Das aber ist das gemeinsame Ziel aller beteiligten Autoren, und deswegen sei ihnen für das Einverständnis, ihre Texte in diesem Kontext zu publizieren, ausdrücklich gedankt.

\section{Bibliographie}

Bobzin, Hartmut (2000): Mohammed. (Beck'sche Reihe 2144, C.H. Beck Wissen). 4. Aufl. 2011. München: C.H. Beck.

Brunner, Rainer (2013): Mohammed. Wissen, was stimmt. (Herder Spektrum 6231). 2. Aufl. Freiburg i. Brsg.: Herder.

Fowden, Garth (2014): Before and After Muhammad: The First Millennium Refocused. Princeton: Princeton University Press.

Jansen, Hans (2008): Mohammed. Eine Biographie. Aus dem Niederländischen übers. von Marlene Müller-Haas. München: C.H. Beck.

Nagel, Tilman (2013): „,Authentizität“ in der Leben-Mohammed-Forschung“. Arabica 60: $516-568$.

Ramadan, Tariq (2009): Muhammad. Auf den Spuren des Propheten. München: Diederichs. Schoeler, Gregor (2011): „Grundsätzliches zu Tilman Nagels Monographie Mohammed. Leben und Legende“. Asiatische Studien 55: 193-209.

4 Schoeler 2011.

5 Nagel 2013. 
Schöller, Marco (2008): Mohammed. (Suhrkamp BasisBiographie 34). Frankfurt a.M.: Suhrkamp.

Shalem, Avinoam (Hg.) (2013): Constructing the Image of Muhammad in Europe. Berlin/Boston: W. de Gruyter.

Tworuschka, Monika (2000): Mohammed. Die Geschichte des Propheten. Düsseldorf: Patmos. 